\title{
CONVOLUTION, FIXED POINT, AND APPROXIMATION \\ IN STIELTJES-VOLTERRA INTEGRAL EQUATIONS
}

CARL W. BITZER

(Received 3 December 1970; revised 10 February 1971)

Communicated by B. Mond

This paper focuses primarily on two aspects of Stieltjes-Volterra integral equation theory. One is a theory for convolution integrals - that is, integrals of the form $\int_{a}^{t} d f[a+t-I] \cdot m$ - and the other is a fixed point theorem for a mapping which is induced by an integral equation. Throughout the paper I will denote the identity function whose range of definition should be clear from the context and all integrals will be left integrals, written $(L) \int_{a}^{b} d f \cdot g$, whose simplest approximating sum is $[f(b)-f(a)] \cdot g(a)$ and whose value is the limit of approximating sums with respect to successive refinements of the interval. Also, $N$ will denote the set of elements of a complete normed ring with unity 1 and $S$ will denote a set linearly ordered by $\leqq$.

In Section 3 the set $S$ denotes all numbers not less than a certain number $a$ and $\leqq$ denotes the natural ordering. A subset $\mathscr{M}$ of the functions from $S$ to $N$ is defined and an integral equation theory is developed for $\mathscr{M}$ with respect to the equation

$$
m(t)=1+(L) \int_{a}^{t} d f[a+t-I] \cdot m .
$$

The ideas for this theory arise in a fairly natural way from the work of Hinton in [3] and my extension of that work in [2]. By a slight extension of the theory in [2] I am able to obtain the results of Section 3 in a reasonably straightforward manner.

The continuation of the theory in [2] is taken up in Section 1. $\mathscr{F}$ denotes a certain class of functions from $S \times S$ to $N$ and $\mathscr{H}$ denotes the reversible function from $\mathscr{F}$ onto $\mathscr{F}$ induced by the integral equation

$$
M(t, x)=1+(L) \int_{x}^{t} d F[t, I] \cdot M[I, x] .
$$

For the definitions and development of that theory the reader is referred to [2] and [3]. He may, however, obtain a fairly good idea of the requirements for 
membership in $\mathscr{F}$ by referring to the proof of Theorem 3.1 in this paper. In Section 2 it is demonstrated that with some restrictions on $N$ the only fixed point of $\mathscr{H}$ is the constant 1 function and that this is true for the expanded theory developed in Section 1 as well. This result is used later in the theory of convolution integrals.

My indebtedness to Professor Hinton in clear. I would also like to express my gratitude both to Professor MacNerney for his encouragement and for raising the fixed point problem in his conversations with me some years ago and to the referee whose careful reading of my paper uncovered several errors and whose recommendations substantially improved its quality.

\section{An extension of $\mathscr{F}$ and $\mathscr{H}$}

If $\{F\}_{0}^{\infty}$ is an $\mathscr{F}$ sequence then the statement that $g$ is a super function for $\{F\}_{0}^{\infty}$ on $[a, b]$ means that $[a, b]$ is an interval of $S, g$ is a non-decreasing function from $[a, b]$ to the numbers, and there is an increasing subdivision $s$ of $(a, b)$ such that if $(x, u)$ and $(x, v)$ are in $\left[s_{p-1}, s_{p}\right] \times\left[s_{p-1}, s_{p}\right]$ and $q$ is a non-negative integer then

$$
\left|F_{q}(x, u)-F_{q}(x, v)\right| \leqq|g(u)-g(v)| .
$$

It is sometimes convenient to describe the foregoing by saying that $g$ is a super function for $\{F\}_{0}^{\infty}$ on $[a, b]$ with respect to $s$. The total variation of a function $f$ on $[a, b]$ will be denoted by $\int_{a}^{b}|d f|$.

Definition 1.1. $\mathscr{G}$ is the set to which $G$ belongs only in case $G$ is the limit of an $\mathscr{F}$ sequence $\{F\}_{0}^{\infty}$ with the following properties:

(i) if $[a, b]$ is an interval of $S$ then there is a super function for $\{F\}_{0}^{\infty}$ on $[a, b]$ and

(ii) if $[a, b]$ is an interval of $S$ and $\varepsilon>0$ then there is a number $n$ such that, for each $x$ in $[a, b]$ and for each integer $p \geqq n$ and each integer $q \geqq n$,

$$
\int_{a}^{b}\left|d\left(F_{p}-F_{q}\right)[x, I]\right|<\varepsilon .
$$

Any $\mathscr{F}$ sequence which satisfies statement (ii) of the foregoing definition converges to some function from $S \times S$ to $N$ and the convergence is uniform on each square of $S \times S$. Consequently, the limit function is uniformly quasi-continuous (the uniform limit of a step function sequence) in its first place on any interval of $S$. In the context of the definition if $[a, b]$ is an interval of $S$ and $\varepsilon>0$ then there is an integer $n$ such that, for each $x$ in $[a, b]$ and each integer $p \geqq n$,

$$
\int_{a}^{b}\left|d\left(F_{p}-G\right)[x, I]\right|<\varepsilon .
$$

Also, if $[a, b]$ is an interval of $S$ then there is a number $K$ such that, for each $x$ in $[a, b]$ and each non-negative integer $p$, 


$$
\int_{a}^{b}\left|d F_{p}[x, I]\right|<K \text { and } \int_{a}^{b}|d G[x, I]|<K .
$$

Moreover, if $g$ is a super function for $\{F\}_{0}^{\infty}$ on $[a, b]$ then $g$ is a super function for $G$ on $[a, b]$.

Using the notation and results of Section 3 it is easy to describe a function which is in $\mathscr{G}$ but not in $\mathscr{F}$. Let $f$ denote a function in $\mathscr{M}$ which has infinitely many points of discontinuity on some interval $[c, d]$ of $S$. Then $T(f)$ is in $\mathscr{G}$ but not in $\mathscr{F}$ since there is no $[a, a, d] T(f)$-chain. See definitions on pages $436-438$ in [2].

LERMMA 1.1. If $[a, b]$ is an interval of $S, g$ is a non-decreasing function from $[a, b]$ to the numbers, $s$ is an increasing subdivision of $(a, b)$, and $K$ is $a$ number greater than 1 then there is a positive number $D$ such that if $F$ is in $\mathscr{F}, g$ is a super function for $F$ on $[a, b]$ with respect to $s, \int_{a}^{b}|d F[t, I]|<K$ for each $t$ in $[a, b], a \leqq x \leqq b, C$ is a non-negative number, and $m$ is $a$ bounded function from $[a, b]$ to the non-negative numbers which satisfies the inequality

$$
m(t) \leqq C+(L) \int_{x}^{t}|d F[t, I]| \cdot m
$$

for each $t$ in $[a, b]$ then

$$
m(t) \leqq C D
$$

for each $t$ in $[a, b]$

Proof. Suppose $[a, b]$ is an interval of $S, g$ is a non-decreasing function from $[a, b]$ to the numbers, $\{s\}_{0}^{n}$ is an increasing subdivision of $(a, b)$, and $K$ is a number greater than 1. Let each of $\{L\}_{0}^{n}$ and $\left\{L^{\prime}\right\}_{0}^{n}$ denote the increasing number sequence defined as follows: (i) $L_{0}^{\prime}=L_{0}=0$ and (ii) if $p$ is a non-negative integer less than $n$ then

$$
L_{p+1}=\left(1+K L_{p}\right) \exp \left\{g\left(s_{p+1}-g\left(s_{p}\right)\right\}\right.
$$

and

$$
L_{p+1}^{\prime}=\left(1+K L_{p}^{\prime}\right) \exp \left\{g\left(s_{n-p}\right)-g\left(s_{n-p-1)}\right\} .\right.
$$

Let $D$ denote $\max \left\{L_{n}, L_{n}^{\prime}\right\}$.

Suppose $F$ is in $\mathscr{F}, g$ is a super function for $F$ on $[a, b]$ with respect to $\{s\}_{0}^{n}, \int_{a}^{b}|d F[t, I]|<K$ for each $t$ in $[a, b], a \leqq x \leqq b, C$ is a non-negative number, and $m$ is a bounded function from $[a, b]$ to the non-negative numbers which satisfies the inequality

$$
m(t) \leqq C+(L) \int_{x}^{t}|d F[t, I]| \cdot m
$$


for each $t$ in $[a, b]$. Let $p$ denote the integer for which $s_{p} \leqq x \leqq s_{p+1}$. If $x \leqq t \leqq s_{p+1}$ then

$$
m(t) \leqq C+(L) \int_{x}^{t} m \cdot d g .
$$

By Theorem 1.4 in [2]

$$
m(t) \leqq C \exp \{g(t)-g(x)\} \leqq C L_{p+1}
$$

for $x \leqq t \leqq s_{p+1}$. Suppose $q$ is a positive integer less than $n-p$ such that $m(t)$ $\leqq C L_{p+q}$ for $x \leqq t \leqq s_{p+q}$. Then, for each $t$ in $\left[s_{p+q}, s_{p+q+1}\right]$,

$$
\begin{aligned}
m(t) & \leqq C+(L) \int_{x}^{s_{p+q}}|d F[t, I]| \cdot m+(L) \int_{s_{p+q}}^{t} m \cdot d g \\
& \leqq C+K C L_{p+q}+(L) \int_{s_{p+q}}^{t} m \cdot d g .
\end{aligned}
$$

Therefore, for each $t$ in $\left[s_{p+q}, s_{p+q+1}\right]$,

$$
\begin{aligned}
m(t) & \leqq\left(C+K C L_{p+q}\right) \exp \left\{g(t)-g\left(s_{p+q}\right)\right\} \\
& \leqq C\left(1+K L_{p+q}\right) \exp \left\{g\left(s_{p+q+1}\right)-g\left(s_{p+q}\right)\right\} \\
& =C L_{p+q+1} .
\end{aligned}
$$

Consequently, $m(t) \leqq C L_{n}$ for $x \leqq t \leqq b$. A similar argument may be employed to show that $m(t) \leqq C L_{n}^{\prime}$ for $a \leqq t \leqq x$. Hence, $m$ is bounded by $C D$ on $[a, b]$.

THEOREM 1.1. if $\{F\}_{0}^{\infty}$ is an $\mathscr{F}$ sequence for which statements (i) and (ii) of Definition 1.1 are true and $\{M\}_{0}^{\infty}$ is the $\mathscr{F}$ sequence defined by $M_{p}=\mathscr{H}\left(F_{p}\right)$ then

(i) $\{M\}_{0}^{\infty}$ converges to a function $H$ from $S \times S$ to $N$ and the convergence is uniform on each square of $S \times S$ and

(ii) if $G=\lim F_{p}$ as $p \rightarrow \infty$ then $H$ is the only function $Q$ from $S \times S$ to $N$ bounded on each square of $S \times S$ such that, for each $(t, x)$ in $S \times S$,

$$
Q(t, x)=1+(L) \int_{x}^{t} d G[t, I] \cdot Q[I, x] .
$$

Proof. Suppose $[a, b]$ is an interval of $S ; \varepsilon>0 ; K$ is a number greater than 1 such that, for each $x$ in $[a, b]$ and each non-negative integer $p$,

$$
\int_{a}^{b}\left|d F_{p}[x, I]\right|<K \text { and } \int_{a}^{b}|d G[x, I]|<K
$$

and $n$ is an integer such that, for each $x$ in $[a, b]$ and for each integer $p \geqq n$ and each nteger $q \geqq \mid \boldsymbol{n}$, 


$$
\int_{a}^{b}\left|d\left(F_{p}-F_{q}\right)[x, I]\right|<\varepsilon .
$$

There is a super function $g$ for $\{F\}_{0}^{\infty}$ on $[a, b]$ with respect to some increasing subdivision $s$ of $(a, b)$. Corresponding to $[a, b], g, s$, and $K$ let $D$ denote a positive number with the property guaranteed by Lemma 1.1. For each non-negative integer $p$ let $k_{p}$ denote the least upper bound for $\left|M_{p}\right|$ on $[a, b] \times[a, b]$. Let each of $p$ and $q$ be an integer not less than $n, x$ be in $[a, b]$ and $m$ be the function from $[a, b]$ to the numbers defined by

$$
m(t)=\left|M_{p}(t, x)-M_{q}(t, x)\right|
$$

Then

$$
\begin{aligned}
m(t) & =\left|(L) \int_{x}^{t} d F_{p}[t, I] \cdot M_{p}[I, x]-(L) \int_{x}^{t} d F_{q}[t, I] \cdot M_{q}[I, X]\right| \\
& \leqq\left|(L) \int_{x}^{t} d\left(F_{p}-F_{q}\right)[t, I] \cdot M_{p}[I, x]\right|+\left|(L) \int_{x}^{t} d F_{q}[t, I] \cdot\left(M_{p}-M_{q}\right)[I, x]\right| \\
& \leqq \varepsilon k_{p}+(L) \int_{x}^{t}\left|d F_{q}[t, I]\right| \cdot m .
\end{aligned}
$$

Using Lemma 1.1 one obtains

$$
m(t) \leqq \varepsilon k_{p} D
$$

for each $t$ in $[a, b]$. In particular

$$
\begin{aligned}
\left|M_{p}(t, x)\right| & \leqq\left|M_{n}(t, x)\right|+\left|M_{n}(t, x)-M_{p}(t, x)\right| \\
& \leqq k_{n}+\varepsilon k_{n} D .
\end{aligned}
$$

Consequently, there is an upper bound $B$ for the sequence $k$ and

$$
\left|M_{p}(t, x)-M_{q}(t, x)\right| \leqq \varepsilon B D
$$

for each $(t, x)$ in $[a, b] \times[a, b]$. Hence, $\{M\}_{0}^{\infty}$ converges to a function $H$ from $S \times S$ to $N$ and the convergence is uniform on each square of $S \times S$. Therefore, the integral

$$
\text { (L) } \int_{x}^{t} d G[t, I] \cdot H[I, x]
$$

exists for each $(t, x)$ in $S \times S$.

Since

$$
\left|H(t, x)-1-(L) \int_{x}^{t} d G[t, I] \cdot H[I, x]\right|
$$




$$
\begin{aligned}
\leqq & \left|H(t, x)-M_{p}(t, x)\right|+\mid(L) \int_{x}^{t} d F_{p}[t, I] \cdot M_{p}[I, x] \\
& -(L) \int_{x}^{t} d G[t, I] \cdot H[I, x] \mid \\
\leqq & \left|H(t, x)-M_{p}(t, x)\right|+\left|(L) \int_{x}^{t} d\left(F_{p}-G\right)[t, I] \cdot M_{p}[I, x]\right| \\
& +\left|(L) \int_{x}^{t} d G[x, I] \cdot\left(M_{p}-H\right)[I, x]\right| \rightarrow 0 \text { as } p \rightarrow \infty \\
H(t, x)= & 1+(L) \int_{x}^{t} d G[t, I] \cdot H[I, x]
\end{aligned}
$$

for each $(t, x)$ in $S \times S$. As in [2] it is clear that $H$ is the only function $Q$ from $S \times S$ to $N$ which is bounded on each square of $S \times S$ such that

$$
Q(t, x)=1+(L) \int_{x}^{t} d G[t, I] \cdot Q[I, x]
$$

for each $(t, x)$ in $S \times S$.

THEOREM 1.2. If $\{F\}_{0}^{\infty}$ is an $\mathscr{F}$ sequence for which statements (i) and (ii) Definition 1.1 are true and $\{M\}_{0}^{\infty}$ is the $\mathscr{F}$ sequence defined by $M_{p}=\mathscr{H}\left(F_{p}\right)$ then

(i) if $[a, b]$ is an interval of $S$ then there is a super function for $\{M\}_{0}^{\infty}$ on $[a, b]$ and

(ii) if $[a, b]$ is an interval of $S$ and $\varepsilon>0$ then there is a number $n$ such that, for each $x$ in $[a, b]$ and for each integer $p \geqq n$ and each integer $q \geqq n$,

$$
\int_{a}^{b}\left|d\left(M_{p}-M_{q}\right)[x, I]\right|<\varepsilon .
$$

Proof. Suppose $[a, b]$ is an interval of $S, g$ is a super function for $\{F\}_{0}^{\infty}$ on $[a, b]$, and $K$ is a number such that $\left|M_{p}\right|=\left|\mathscr{H}\left(F_{p}\right)\right|<K$ on $[a, b] \times[a, b]$ for each non-negative integer $p$. Let $h$ denote the function from $[a, b]$ to the numbers defined by

$$
h(t)=g(t) K \exp \{g(b)-g(a)\}
$$

From the proof of Theorem 3.2 in [2] it is clear that if $p$ is a non-negative integer and $g$ is a super function for $F_{p}$ on $[a, b]$ with respect to a subdivision $s$ of $(a, b)$ then $h$ is a super function for $M_{p}$ on $[a, b]$ with respect to the same subdivision $s$. Therefore, $h$ is a super function for $\{M\}_{0}^{\infty}$ on $[a, b]$.

Now suppose $[a, b]$ is an interval of $S, F$ and $G$ are in $\mathscr{F}, M=\mathscr{H}(F), H=\mathscr{H}(G)$ $\varepsilon>0,|M-H|<\varepsilon$ on $[a, b] \times[a, b]$, 


$$
\int_{a}^{b}|d(F-G)[x, I]|<\varepsilon
$$

for each $x$ in $[a, b]$, and $K$ is a number greater than 1 such that $|M|<K$ on $[a, b] \times[a, b]$ and each of $\int_{a}^{b}|d G[t, I]|$ and $\int_{a}^{b}|d M[t, I]|$ is less than $K$ for each $t$ in $[a, b]$. There is a super function $g$ for $G$ on $[a, b]$ with respect to some increasing subdivision $v$ of $(a, b)$. Corresponding to $[a, b], g, v$, and $K$ let $D$ denote a positive number with the property guaranteed by Lemma 1.1. Let $x$ be a member of $S$ such that $a<x \leqq b$ and let $\{s\}_{0}^{n}$ be a subdivision of $(a, x)$. Let $m$ denote the function from $[a, x]$ to the numbers such that $m(a)=0$ and if $a<t \leqq x, u_{k}=t$, and $\{u\}_{0}^{k-1}$ is the common part of $s$ and $[0, n] \times[a, t]$ then

$$
m(t)=\sum_{p=1}^{k}\left|M\left(t, u_{p}\right)-H\left(t, u_{p}\right)-\left\{M\left(t, u_{p-1}\right)-H\left(t, u_{p-1}\right)\right\}\right| .
$$

Suppose $t$ and $u$ are as in the foregoing.

Then

$$
\begin{aligned}
m(t) & =\sum_{p=1}^{k} \mid(L) \int_{u_{p}}^{t} d F[t, I] \cdot M\left[I, u_{p}\right] \\
& -(L) \int_{u_{p}}^{t} d G[t, I] \cdot H\left[I, u_{p}\right]-\left\{(L) \int_{u_{p}}^{t} d F[t, I] \cdot M\left[I, u_{p-1}\right]\right. \\
& \left.-(L) \int_{u_{p}}^{t} d G[t, I] \cdot H\left[I, u_{p-1}\right]\right\}-\left\{(L) \int_{u_{p}-1}^{u_{p}} d F[t, I] \cdot M\left[I, u_{p-1}\right]\right. \\
& \left.-(L) \int_{u_{p-1}}^{u} d G[t, I] \cdot H\left[I, u_{p-1}\right]\right\} \mid
\end{aligned}
$$

Since

$$
\begin{aligned}
\sum_{p=1}^{k} & \left\{(L) \int_{u_{p}-1}^{u_{p}}|d(F-G)[t, I]| \cdot\left|M\left[I, u_{p-1}\right]\right|\right. \\
+ & (L) \int_{u_{p}-1}^{u_{p}}|d G[t, I]| \cdot \mid(M-H)\left[I, u_{p-1} \mid\right\}<2 \varepsilon K \\
m(t) & <2 \varepsilon K+\sum_{p=1}^{k} \mid(L) \int_{u_{p}}^{t} d F[t, I] \cdot\left\{M\left[I, u_{p}\right]-M\left[I, u_{p-1}\right]\right\} \\
& -(L) \int_{u_{p}}^{t} d G[t, I] \cdot\left\{H\left[I, u_{p}\right]-H\left[I, u_{p-1}\right]\right\} \mid \\
& =2 \varepsilon K+\sum_{p=1}^{k} \mid(L) \int_{u_{p}}^{t} d(F-G)[t, I] \cdot\left\{M\left[I, u_{p}\right]-M\left[I, u_{p-1}\right]\right\} \\
& +(L) \int_{u_{p}}^{t} d G[t, I] \cdot\left\{(M-H)\left[I, u_{p}\right]-(M-H)\left[I, u_{p-1}\right]\right\} \mid
\end{aligned}
$$




$$
\begin{aligned}
& \leqq 2 \varepsilon K+(L) \int_{a}^{t}|d(F-G)[t, I]| \cdot \sum_{p=1}^{k}\left|M\left[I, u_{p}\right]\right|-M\left[I, u_{p-1}\right] \\
& +(L) \int_{a}^{t}|d G[t, I]| \cdot \sum_{p=1}\left|(M-H)\left[I, u_{p}\right]-(M-H)\left[I, u_{p-1}\right]\right| \\
& <3 \varepsilon K+(L) \int_{a}^{t}|d G[t, I]| \cdot m .
\end{aligned}
$$

Therefore, by Lemma 1.1

$$
m(t) \leqq 3 \varepsilon K D
$$

for each $t$ in $[a, x]$. Consequently,

$$
\sum_{s}|d(M-H)[x, I]| \leqq 3 \& K D
$$

for each $x$ in $(a, b]$ and each subdivision $s$ of $(a, x)$. A similar result may be obtained for subdivisions of $(x, b)$ when $a \leqq x<b$.

In the proof of Theorem 3.3 in [2] one observes that the existence of a bound for $\int_{a}^{b}|d M[t, I]|, a \leqq t \leqq b$, depends only on the existence of super functions for $F$ and $M$, the bound for $M$ on $[a, b] \times[a, b]$, and the bound for $\int_{a}^{b}|d F[t, I]|, a \leqq t \leqq b$. This observation together with the foregoing results suffice to establish an argument for part (ii) of the conclusion.

THEOREM 1.3. If each of $G$ and $H$ is in $\mathscr{G}$ and

$$
H(t, x)=1+(L) \int_{x}^{t} d G[t, I] \cdot H[I, x]
$$

for each $(t, x)$ in $S \times S$, then

$$
G(t, x)=1+(L) \int_{x}^{t} d H[t, I] \cdot G[I, x]
$$

for each $(t, x)$ in $S \times S$.

This is an immediate consequence of Theorem 1.1 and 1.2 and the fact that $\mathscr{H}(\mathscr{H}(F))=F$.

Observe that if $f$ is a super function for $F$ on $[a, b]$ with respect to a subdivision $s$ of $(a, b)$ and $g$ is a super function for $G$ on $[a, b]$ with respect to a subdivison $t$ of $(a, b)$ then there is a common refinement $r$ of $s$ and $t$ such that $f+g$ is a super function for $F$ and $G$ on $[a, b]$ with respect to $r$. This observation together with the derivation of the non-homogeneous equation from the homogeneous equation as in [2] yields the analogues for $\mathscr{G}$ of Theorems 4.1 and 4.2 in [2]. In particular we have 
THEOREM 1.4. If each of $E$ and $F$ is in $\mathscr{G}$ then there is only one function $P$ from $S \times S$ to $N$, bounded on each square of $S \times S$, such that, for each $(t, x)$ in $S \times S$,

$$
P(t, x)=E(t, x)+(L) \int_{x}^{t} d F[t, I] \cdot P[I, x] .
$$

Moreover, $P$ is in $\mathscr{G}$.

\section{A fixed point theorem for $\mathscr{H}$}

Notice that $\mathscr{F}$ is a subset of $\mathscr{G}$. In the remainder of this paper it will be convenient to regard $\mathscr{H}$ as being extended to include all of $\mathscr{G}$ in its range of definition subject to the condition that $(F, M)$ is in $\mathscr{H}$ only in case

$$
M(t, x)=1+(L) \int_{x}^{t} d F[t, I] \cdot M[I, x]
$$

for each $(t, x)$ in $S \times S$. From Theorems $1.1,1.2$, and 1.3 it is clear that $\mathscr{H}$ is a reversible function from $\mathscr{G}$ onto $\mathscr{G}$ and that $\mathscr{H}(\mathscr{H}(F))=F$ for each $F$ in $\mathscr{C}$.

Suppose $M=\mathscr{H}(F),[c, d]$ is an interval of $S$, and $g$ is a super function for $F$ on $[c, d]$ such that if each of $(x, u)$ and $(x, v)$ is in $[c, d] \times[c, d]$ then $\mid F(x, u)$ $-F(x, v)|\leqq| g(u)-(v) \mid$. By examining the proof of Hinton's approximation theorem on pages 327 and 328 of [3] one observes that it may be applied to the restriction of $F$ to $[c, d] \times[c, d]$. Consequently, if $(a, b)$ is in $[c, d] \times[c, d], K$ is an upper bound for $|M[I, a]|$ on $[c, d], s$ is a $(g-M[I, a]-\varepsilon)$ chain from $a$ to $b$ (see [2] and [3]), $\{r\}_{0}^{m}$ is a refinement of $s$,

$$
\varepsilon^{\prime}=2 \varepsilon(K+|g(b)-g(a)|),
$$

and $\{z\}_{0}^{m}$ is the $N$ sequence defined inductively by $z_{0}=1$ and

$$
z_{p}=1+\sum_{q=1}^{p}\left[F\left(r_{p}, r_{q}\right)-F\left(r_{p}, r_{q-1}\right)\right] \cdot z_{q-1},
$$

then

$$
\left|z_{m}-M(b, a)\right| \leqq \varepsilon^{\prime} \prod_{q=1}^{m}\left(1+\left|g\left(r_{q}\right)-g\left(r_{q-1}\right)\right|\right) .
$$

In general if $F$ is in $\mathscr{F}$ and $[c, d]$ is an interval of $S$ then it is only required that there be a super function for $F$ on $[c, d]$ with respect to some increasing subdivision of $(c, d)$. In the foregoing it is assumed that there is a super function for $F$ on $[c, d]$ with respect to the subdivision $\{s\}_{0}^{1}$, that is $s_{0}=c$ and $s_{1}=d$. To see that the approximation theorem does not apply without restriction to members of $\mathscr{F}$ consider the following example. Let $T$ denote the subset of $[0,2] \times[0,2]$ to which $(x, y)$ belongs only in case (i) $0<x \leqq 2$ and $y=0$ or (ii) $x=2$ and $0 \leqq y<2$ 
or (iii) $x>1$ and $y<2-x$. Let $F$ denote the function from $[0,2] \times[0,2]$ to the numbers defined by $F(x, y)=0$ if $(x, y)$ is in $T$ and $F(x, y)=1$ otherwise. Suppose $S$ is $[0,2]$ and $N$ is the set of numbers. Then $F$ is in $\mathscr{F}$ and we may let $M$ denote $\mathscr{H}(F)$. Observe that $M[I, 0]$ is 2 on $(0,1], 3$ on $(1,2)$, and 4 at 2 . However, for every subdivision of $(0,2)$ there is a refinement $\{r\}_{0}^{m}$ such that with respect to it the approximation for $M$ at $(2,0)$ is 3 . This may be accomplished by choosing $r$ so that $r_{m}-r_{m-1}=r_{1}-r_{0}<1$.

Now suppose $S$ is $[0,1]$ and $F$ is the function from $S \times S$ to $N$ defined by $F(x, y)=0$ when $(x, y)=(1,0)$ and $F(x, y)=1$ otherwise. Notice that if the unit of $N$ satisfied the equation $1+1=0$ then $\mathscr{H}(F)=F$. If, however, the unit of $N$ is different from 0 and there is a number $h>1$ such that $h|x| \leqq|x+x|$ for each $x$ in $N$ then the equation $1+1=0$ does not hold and $F$ is not a fixed point of $\mathscr{H}$. The remainder of this section is devoted to showing that under the foregoing conditions the only fixed point of $\mathscr{H}$ is the constant 1 function.

LEMMA 2.1. If $k \geqq 0, h>1,\{d\}_{0}^{m}$ is a non-negative number sequence with $d_{0}=1$, and $\{D\}_{0}^{m}$ is a non-negative number sequence such that

$$
D_{p} \leqq k \prod_{q=1}^{p}\left(1+d_{q}\right)+(1 / h) \sum_{q=2}^{p} d_{q} D_{q-1}
$$

for every non-negative integer $p$ not greater than $m$; then

$$
D_{p} \leqq k \prod_{q=1}^{p}\left(1+d_{q}\right) \sum_{q=0}^{p} d_{q}
$$

for every non-negative integer $p$ not greater than $m$.

Observe that it suffices to prove the restatement of Lemma 2.1 obtained by replacing the inequality in the hypothesis with equality.

THEOREM 2.1. If there is a number $h>1$ such that $h|x| \leqq|x+x|$ for each $x$ in $N, F=\mathscr{H}(F),[c, d]$ is an interval of $S$, and $g$ is a super function for $F$ on $[c, d]$ such that if $(x, u)$ and $(x, v)$ are in $[c, d] \times[c, d]$ then $|F(x, u)-F(x, v)|$ $\leqq|g(u)-g(v)| ;$ then $F$ is the constant 1 function on $[c, d] \times[c, d]$.

Proof. Suppose that $N, h, F,[c, d]$, and $g$ are as in the hypothesis of the theorem and that $[a, b]$ is a subinterval of $[c, d]$. The proof will consist of showing that $F(b, a)=1$. The argument that $F(a, b)=1$ is similar.

It will be assumed that the unit of $N$ is different from 0 since in that case it is the only member of $N$ and the conclusion is immediate. Let $\{s\}_{0}^{2 n}$ be a $(g-F[I, a]-\varepsilon)$-chain from $a$ to $b, K$ be an upper bound for $|F[I, a]|$ on $[a, b]$ and

$$
\varepsilon^{\prime} h /(h+1)=2 \varepsilon(K+|g(b)-g(a)|) .
$$

Define the $N$ sequence $\{z\}_{0}^{2 n}$ inductively by $z_{0}=1$ and 


$$
z_{p}=1=\sum_{q=1}^{p}\left[F\left(s_{p}, s_{q}\right)-F\left(s_{p}, s_{q-1}\right)\right] \cdot z_{q-1} .
$$

Define the number sequence $\{d\}_{0}^{2 n}$ by $d_{0}=1$ and $d_{p}=\left|g\left(s_{p}\right)-g\left(s_{p-1}\right)\right|$ for $0<p$ $\leqq 2 n$. In the proof of Theorem 5.1 in [3] Hinton establishes the following inequality for every integer $p$ in $[0,2 n]$ :

$$
\varepsilon^{\prime}[h /(h+1)] \prod_{q=1}^{p}\left(1+d_{q}\right) \geqq\left|z_{p}-F\left(s_{p}, a\right)\right| .
$$

Therefore,

$$
\begin{aligned}
& \varepsilon^{\prime}[h /(h+1)] \prod_{q=1}^{p}\left(1+d_{q}\right) \\
& \geqq \mid 1+\sum_{q=1}^{p}\left[F\left(s_{p}, s_{q}\right)-F\left(s_{p}, s_{q-1}\right)\right] \cdot\left[z_{q-1}-1\right] \\
& \quad+\sum_{q=1}^{p}\left[F\left(s_{p}, s_{q}\right)-F\left(s_{p}, s_{q-1}\right)\right]-F\left(s_{p}, a\right) \mid \\
& \geqq h\left|1-F\left(s_{p}, a\right)\right|-\sum_{q=1}^{p} d_{q}\left|z_{q-1}-1\right|
\end{aligned}
$$

Consequently,

$$
\begin{aligned}
\left|1-F\left(s_{p}, a\right)\right| \leqq & {\left[\varepsilon^{\prime} /(h+1)\right] \prod_{q=1}^{p}\left(1+d_{q}\right) } \\
& +(1 / h) \sum_{q=2}^{p} d_{q}\left|z_{q-1}-1\right|
\end{aligned}
$$

and

$$
\begin{aligned}
\left|z_{p}-1\right| & \leqq\left|z_{p}-F\left(s_{p}, a\right)\right|+\left|F\left(s_{p}, a\right)-1\right| \\
& \leqq \varepsilon^{\prime} \prod_{q=1}^{p}\left(1+d_{q}\right)+(1 / h) \sum_{q=2}^{p} d_{q}\left|z_{q}-1\right|
\end{aligned}
$$

for every non-negative integer $p$ not greater than $2 n$. By using Lemma 2.1 together with the approximation theorem stated earlier the following inequality may be established.

$$
\begin{aligned}
|1-F(b, a)| \leqq & 1-z_{2 n}|+| z_{2 n}-F(b, a) \mid \\
\leqq & \varepsilon^{\prime} \prod_{q=1}^{2 n}\left(1+d_{q}\right) \sum_{q=0}^{2 n} d_{q} \\
& \quad+\varepsilon^{\prime}[h /(h+1)] \prod_{q=1}^{2 n}\left(1+d_{q}\right)
\end{aligned}
$$




$$
\begin{aligned}
& =\varepsilon^{\prime}\{1+|g(b)-g(a)|+h /(h+1)\} \prod_{q=1}^{2 n}\left(1+d_{q}\right) \\
& \leqq \varepsilon^{\prime}\{2+|g(b)-g(a)|\} \exp |g(b)-g(a)| .
\end{aligned}
$$

This completes the proof.

Now suppose that $N$ and $h$ are as in Theorem 2.1. Notice that if $x$ is in $N$ then $x+x=0$ only in case $x=0$. Suppose $[a, b]$ is an interval of $S, F$ is a function from $S \times S$ to $N$ such that

$$
F(t, x)=1+(L) \int_{x}^{t} d F[t, I] \cdot F[I, x]
$$

for each $(t, x)$ is $S \times S$, and $\{s\}_{0}^{n}$ is an increasing subdivision of $(a, b)$ such that $F$ is the constant 1 function on $\left[s_{p-1}, s_{p}\right] \times\left[s_{p-1}, s_{p}\right]$ for each counting number $p$ not greater than $n$. If

$$
s_{0} \leqq x \leqq s_{1} \leqq t \leqq s_{2}
$$

then

$$
\begin{aligned}
F(t, x) & =1+(L) \int_{x}^{s_{1}} d F[t, I] \cdot 1+(L) \int_{s_{1}}^{t} d 1 \cdot F[I, x] \\
& =1+F\left(t, s_{1}\right)-F(t, x) \\
& =1+1-F(t . x)
\end{aligned}
$$

or

$$
[F(t, x)-1]+[F(t, x)-1]=0 .
$$

Similarly if $s_{0} \leqq t \leqq s_{1} \leqq x \leqq s_{2}$ then $F(t, x)=1$. Consequently, one may use an inductive argument to show that $F$ is 1 on $[a, b] \times[a, b]$. Using these observations in conjunction with Theorem 2.1 we have

THEOREM 2.2. If there is a number $h>1$ such that $h|x| \leqq|x+x|$, for each $x$ in $N$, and $F=\mathscr{H}(F)$ then $F$ is the constant 1 function on $S \times S$.

\section{Convolution integrals}

Let $a$ denote a number and let $S$ denote $[a, \infty)$. If $f$ is a function from $S$ to $N$ then $T^{\prime}(f)$ will denote the function from $S \times S$ to $N$ defined by

$$
T(f)(x, y)=\left\{\begin{array}{cc}
f(a+x-y) & \text { when } y \leqq x) \\
1 & \text { when } x<y .
\end{array}\right.
$$

$\mathscr{M}$ is the set to which $f$ belongs only in case $f$ is a function from $S$ to $N, f(a)=1, f$ is of bounded variation on every interval $[a, b]$ of $S$, and there is a number $c>a$ 
such that $f$ satisfies a uniform Lipschitz condition on $[a, c]$ (see [1] page 257). $\mathscr{M}^{\prime}$ will denote the subset of $\mathscr{M}$ to which $f$ belongs only in case $f$ has at most finitely many points of discontinuity.

THEOREM 3.1. If $f$ is in $\mathscr{M}^{\prime}$ then $T(f)$ is in $\mathscr{F}$.

Proof. Suppose $f$ is in $\mathscr{M}^{\prime}$ and $F=T(f)$. Clearly $F(x, x)=1$ for each $x$ in $S$. Suppose $b$ is a number greater than $a$. If $x$ is in $[a, b]$ then

$$
\int_{a}^{b}|d F[x, I]|=\int_{a}^{x}|d f[a+x-I]|=\int_{a}^{x}|d f| .
$$

Therefore, $1+\int_{a}^{b}|d f|$ is a number $K$ such that $\int_{a}^{b}|d F[x, I]|<K$ for each $x$ in $[a, b]$. Suppose $x$ is in $S$. Then $F[I, x]$ is of bounded variation on $[a, b]$ and, hence, uniformly quasi-continuous on $[a, b]$, that is, the uniform limit of a step function sequence. Since $F(t, x+)=1$ when $a \leqq t \leqq x$ and $F(t, x+)=\lim$ $f(a+t-h)$ as $h \rightarrow x+$ when $t>x, F[I, x+]$ is uniformly quasi-continuous on $[a, b]$. Similarly, if $x>a$ then $F[I, x-]$ is uniformly quasi-continuous on $[a, b]$. Since $f$ satisfies a uniform Lipschitz condition on an interval $[a, c]$ there are positive numbers $\delta$ and $M$ such that $\delta<c-a$ and if $u$ and $v$ are in $[a, c]$ and $|u-v|<\delta$ then $|f(u)-f(v)|<M|u-v|$. If $s$ is an increasing subdivision of $(a, b),|s|<\delta$, and each of $(w, u)$ and $(w, v)$ is in

$$
\begin{aligned}
& {\left[s_{p-1}, s_{p}\right] \times\left[s_{p-1}, s_{p}\right] \text { then }} \\
& \text { (i) }|F(w, u)-F(w, v)| \\
& =|f(a+w-u)-f(a+w-v)| \\
& <M|u-v| \text { when } u \leqq w \text { and } v \leqq w, \\
& \text { (ii) }|F(w, u)-F(w, v)| \\
& =|F(w, w)-F(w, v)| \\
& =|f(a)-f(a+w-v)| \\
& <M|v-w| \leqq M|v-u| \\
& \text { when } v \leqq w \leqq u, \text { and } \\
& \text { (iii) }|F(w, u)-F(w, v)| \\
& =|1-1|=0 \leqq M|v-u| \\
& \text { when } w \leqq u \text { and } w \leqq v .
\end{aligned}
$$

Therefore, the function $g$ from $[a, b]$ to the numbers defined by $g(t)=M t$ is a super function for $F$ on $[a, b]$.

Now suppose $a \leqq x \leqq b$ and $s$ is an increasing subdivision of $(a, b)$ whose 
final set consists of $a, x, b$, and all numbers in $[a, b]$ at which $F[I, x]$ is discontinuous. To see that $s$ is an $[a, x, b] F$-chain (see [2]) consider the following. Suppose $x \leqq s_{p-1}<r \leqq t<s_{p}$. Let $c$ and $d$ be numbers such that $s_{p-1}<c<r$ $\leqq t<d<s_{p}$ and let $\delta^{\prime}=\min \{r-c, d-t\}$. Since $f[a+I-x]=F[I, x]$ on $[c, d]$ and $F[I, x]$ is continuous and of bounded variation on $[c, d]$, the variation function $h$ defined by

$$
h(t)=\int_{c}^{t}|d f[a+I-x]|
$$

is continuous on $[c, d]$, hence, uniformly continuous on $[c, d]$. Let $\varepsilon$ be a positive number. There is a positive number $\delta^{\prime \prime}$ such that if $c \leqq u \leqq v \leqq d$ and $v-u<3 \delta^{\prime \prime}$ then

$$
\int_{u}^{v}|d f[a+I-x]|=h(v)-h(u)<\varepsilon .
$$

Let $\delta=\min \left\{\delta^{\prime}, \delta^{\prime \prime}\right\}$. Suppose $r \leqq w \leqq t$ and either $x-\delta \leqq u \leqq v<x$ or $x<u$ $\leqq v \leqq x+\delta$.

Then

$$
\begin{aligned}
\int_{u}^{v}|d F[w, I]| & \leqq \int_{x-\delta}^{x+\delta}|d F[w, I]| \\
& =\int_{x-\delta}^{x+\delta}|d f[a+w-I]| \\
& =\int_{w-\delta}^{w+\delta}|d f[a+I-x]|<\varepsilon
\end{aligned}
$$

since $c \leqq w-\delta<w+\delta \leqq d$ and $2 \delta<3 \delta^{\prime \prime}$. This completes the proof.

Theorem 3.2. If $g$ is in $\mathscr{M}$ then $T(g)$ is in $\mathscr{G}$.

Proof. Suppose $g$ is in $\mathscr{M}$ and $\{d\}_{0}^{\infty}$ is a reversible $S$ sequence whose final set contains every number at which $g$ is discontinuous. Let $L$ denote the function from $S$ to $N$ defined by $L(a)=0$ and $L(x)=g(x)-\lim g(t)$ as $t \rightarrow x-$ and let $R$ denote the function from $S$ to $N$ defined by $R(x)=\lim g(t)-g(x)$ as $t \rightarrow x+$. Define the $\mathscr{M}^{\prime}$ sequence $\{f\}_{0}^{\infty}$ by

$$
f_{p}(x)=\left\{\begin{array}{clrl}
0 & \text { when } a \leqq x<d_{p} \\
L(x) & \text { when } x & =d_{p} \\
L(x)+R(x) & \text { when } x & >d_{p} .
\end{array}\right.
$$

The sequence of partial sums $\Sigma f$ converges to a function $h$ which is of bounded variation on each interval of $S$. Moreover, $g-h$ is continuous and 


$$
\lim _{a}^{b}\left|d\left(h-\sum_{0}^{n} f\right)\right| \rightarrow 0 \text { as } n \rightarrow \infty
$$

for each $b$ in $S$. Let $\{F\}_{0}^{n}$ denote the $\mathscr{F}$ sequence defined by

$$
F_{n}=T\left(g-h+\sum_{0}^{n} f\right)
$$

and let

$$
G=\lim F_{n} \text { as } n \rightarrow \infty \text {. }
$$

Evidently, $G$ is in $\mathscr{G}$ and

$$
\begin{aligned}
T(g) & =T\left(\lim _{n \rightarrow \infty} F_{n}[I, a]\right) \\
& =\lim _{n \rightarrow \infty} T\left(F_{n}[I, a]\right) \\
& =\lim _{n \rightarrow \infty} F_{n}=G .
\end{aligned}
$$

TH:EOREM 3.3. If $f$ is a function from $S$ to $N$ such that $T(f)$ is in $\mathscr{G}$ then $f$ is in $\mathscr{M}$.

Proof. Suppose $f$ is a function from $S$ to $N$ such that $T(f)$ is in $\mathscr{G}$. Let $F$ denote $T(f)$. Then $1=F(a, a)=f(a)$. If $b>a$ then

$$
\int_{a}^{b}|d F[b, I]|=\int_{a}^{b}|d f[a+b-I]|=\int_{a}^{b}|d f| .
$$

Therefore, $f$ is of bounded variation on each interval of $S$.

Let $g$ denote a non-decreasing super function for $F$ on $[a, d]$ such that if each of $u, v$, and $w$ is in $[a, d]$ then

$$
|F(w, u)-F(w, v)| \leqq|g(u)-g(v)| .
$$

Let $c=(a+d) / 2$ and suppose that $f$ does not satisfy a uniform Lipshitz condition on $[a, c]$. Then for every number $K$ there are numbers $u$ and $v$ in $[a, c]$ such that

$$
|f(u)-f(v)|>K|u-v| \text {. }
$$

Let $K=[g(d)-g(a)] /(c-a)$ and $(u, v)$ be a number pair such that $a \leqq u<v \leqq c$ and $|f(u)-f(v)|>K|u-v|$. Let $n$ denote a counting number such that

$$
n|u-v| \leqq d-u<(n+1)|u-v|
$$

Note that $c-a \leqq n|u-v|$. For each counting number $p$ not greater than $n$,

$$
g(a+p|u-v|)-g(a+(p-1)|u-v|)
$$




$$
\begin{aligned}
\geqq & \mid F(u+p|u-v|, a+p|u-v|) \\
& -F(u+p|u-v|, a+(p-1)|u-v|) \mid \\
= & |f(u)-f(v)|>K|u-v| .
\end{aligned}
$$

Therefore,

$$
\begin{aligned}
g(a & +n|u-v|)-g(a) \\
& =\sum_{p=1}^{n}[g(a+p|u-v|)-g(a+(p-1)|u-v|)] \\
& >n K|u-v| \geqq g(d)-g(a)
\end{aligned}
$$

which is impossible since $a+n|u-v| \leqq d$. This completes the proof.

THEOREM 3.4. If $f$ is in $\mathscr{M}$ then there is only one function $m$ from $S$ to $N$ bounded on each interval of $S$ such that

$$
m(t)=1+(L) \int_{a}^{t} d f[a+t-I] \cdot m
$$

for each $t$ in $S$. Moreover, $m$ is in $\mathscr{M}$.

Proof. Suppose $f$ is in $\mathscr{M}$. Let $F$ denote $T(f)$ and let $M=\mathscr{H}(F)$. Define the function $m$ from $S$ to $N$ by $m(t)=M(t, a)$. Then $m$ is bounded on each interval of $S$ and, for each $t$ in $S$,

$$
\begin{aligned}
m(t) & =M(t, a)=1+(L) \int_{a}^{t} d F[t, I] \cdot M[I, a] \\
& =1+(L) \int_{a}^{t} d f[a+t-I] \cdot m .
\end{aligned}
$$

Suppose $p$ is a function from $S$ to $N$ bounded on each interval of $S$ such that

$$
p(t)=1+(L) \int_{a}^{t} d f[a+t-I] \cdot p
$$

for each $t$ in $S$. Let $P$ denote $T(p)$. Note that $P$ is bounded on each square of $S \times S$. If $a \leqq t \leqq x$ then

$$
\text { (L) } \int_{x}^{t} d F[t, I] \cdot P[I, x]=0=P(t, x)-1 .
$$

If $a \leqq x<t$ then

$$
\text { (L) } \int_{x}^{t} d F[t, I] \cdot P[I, x]
$$




$$
\begin{aligned}
& =(L) \int_{x}^{t} d f[a+t-I] \cdot p[a+I-x] \\
& =(L) \int_{a}^{a+t-x} d f[2 a+t-x-I] \cdot p \\
& =p(a+t-x)-1=P(t, x)-1 .
\end{aligned}
$$

By Theorem 1.3 we have that $P=\mathscr{H}(F)=M$ and, consequently, $p=m$. From the foregoing it is clear that $M=T(m)$. Therefore, $m$ is in $\mathscr{M}$.

The next theorem may be validated by recalling that if $M=\mathscr{H}(F)$ then $F=\mathscr{H}(M)$ and that if $F=T(f)$ then $\mathscr{H}(F)=T(m)$.

THEOREM 3.5. If $f$ is a function in $\mathscr{M}$ and $m$ is the unique function in $\mathscr{M}$ which satisfies the equation

$$
m(t)=1+(L) \int_{a}^{t} d f[a+t-I] \cdot m,
$$

for each $t$ in $S$, then

$$
f(t)=1+(L) \int_{a}^{t} d m[a+t-I] \cdot f
$$

for each $t$ in $S$.

By employing 1.4 Theorem and arguments similar to those used for Theorem 3.4 there is no difficulty in validating the next theorem.

THEOREM 3.6. If each of $f$ and $e$ is in $\mathscr{M}$ then there is only one function $p$ from $S$ to $N$ bounded on each interval of $S$ such that

$$
p(t)=e(t)+(L) \int_{a}^{t} d f[a+t-I] \cdot p
$$

for each $t$ in $S$. Moreover, $p$ is in $\mathscr{M}$.

THEOREM 3.7. Iff is a continuous function in $\mathscr{M}$ and $m$ is the unique function in $\mathscr{M}$ which satisfies the equation

$$
m(t)=1+(L) \int_{a}^{t} d f[a+t-I] \cdot m,
$$

for each $t$ in $S$, then $m$ is continuous.

Proof. Suppose each of $f$ and $m$ is as in the hypothesis of the theorem. Let $F=T(f)$ and $M=\mathscr{H}(F)$. Then $M=T(m)$. Since $f$ is continuous $F$ is in $\mathscr{F}$ and $F$ is continuous with respect to its first place, in fact, $F$ is continuous. By Theorem 5.6 in [2], $M$ is continuous with respect to its first place. Therefore, $m$ is con- 
tinuous. The next theorem may be obtained in a similar manner by using Theorem 5.7 in [2].

THEOREM 3.8. If each of $f$ and $e$ is a continuous function in $\mathscr{M}$ and $p$ is the unique function in $\mathscr{M}$ such that

$$
p(t)=e(t)+(L) \int_{a}^{t} d f[a+t-I] \cdot p
$$

for each $t$ in $S$, then $p$ is continuous.

THEOREM 3.9. If there is a number $h>1$ such that $h|x| \leqq|x+x|$, for each $x$ in $N$, and $f$ is a function in $\mathscr{M}$ such that

$$
f(t)=1+(L) \int_{a}^{t} d f[a+t-I] \cdot f,
$$

for each $t$ in $S$, then $f$ is the constant 1 function on $S$.

Proof. Suppose $f$ satisfies the hypothesis of the theorem. Let $F=T(f)$. If $a \leqq x<t$ then

$$
\begin{aligned}
(L) \int_{x}^{t} d F[t, I] \cdot F[I, x] & =(L) \int_{x}^{t} d f[a+t-I] \cdot f[a+I-x] \\
& =(L) \int_{a}^{a+t-x} d f[2 a+t-x-I] \cdot f \\
& =f(a+t-x)-1=F(t, x)-1 .
\end{aligned}
$$

Therefore, $\mathscr{H}(F)=F$ and by Theorem $2.2, T(f)=F=1$. Consequently, $f$ is the constant 1 function on $S$.

\section{References}

[1] T. M. Apostol, Mathematics Analysis. (Addison-Wesley, Reading, 1957).

[2] C. W. Bitzer, 'Stieltjes-Volterra Integral Equations', Illinois J. Math. 14 (1970), 434-451.

[3] D. B. Hinton, 'A Stieltjes-Volterra Integral Equation Theory', Canad. J. Math. 18 (1966), 314-331.

The Department of Mathematics

The University of North Carolina

Greensboro

U.S.A. 